\title{
A Three-Dimensional Study of Body Motion During Ergometer Rowing
}

\author{
Chiarella Sforza $^{*}, 1$, Elena Casiraghi ${ }^{1,2}$, Nicola Lovecchio ${ }^{1}$, Domenico Galante ${ }^{1}$ and Virgilio F. Ferrario ${ }^{1}$ \\ ${ }^{1}$ Laboratorio di Anatomia Funzionale dell'Apparato Locomotore (LAFAL), Functional Anatomy Research Center \\ (FARC), Dipartimento di Morfologia Umana e Scienze Biomediche "Città Studi", Facoltà di Medicina e Chirurgia, \\ Università degli Studi di Milano, via Mangiagalli 31, 20133 Milano, Italy \\ ${ }^{2}$ Dottorato di Ricerca in Scienze dello Sport, Facoltà di Scienze Motorie, Università degli Studi di Milano, via Kramer \\ 4/A, 20129 Milano, Italy
}

\begin{abstract}
Background: Rowing movements can be simulated using specialized ergometers; the method can be used both for training and indoor assessment of body movements within controlled conditions.

Purpose: To perform a three-dimensional quantitative analysis of body movements during ergometer rowing, and to examine if there is a relationship between anthropometry and rowing kinematics.

Study Design: Descriptive Laboratory Study

Methods: Body movements were recorded in 18 high-level oarsmen during ergometer rowing at 28 strokes/min. The three-dimensional movements of 21 body landmarks (left and right ankle, knee, greater trochanter, hip, shoulder, elbow, wrist, tragus; spinous process of $\mathrm{C} 7, \mathrm{~T} 2, \mathrm{~T} 12, \mathrm{~L} 2, \mathrm{~L} 4)$ were detected by an optoelectronic instrument. Using dedicated software, the range of motion of the posterior angles of the cervical, thoracic and lumbar spine segments relative to the horizontal axis; the angles between greater trochanter- knee- ankle and between knee-ankle and the ground; head rotation and tilt; leg and upper limb symmetry, were computed.

Results: The head and neck were approximately in line with the horizontal at catch, and extended at finish, with limited horizontal and frontal plane inclinations. Thoracic spine extension during the stroke was on average $68^{\circ}$. Lumbar spine range of motion was on average $59^{\circ}$, and it was smaller in weightier oarsmen. Upper limbs were symmetric, and a complete, symmetric extension of the lower limbs was made. At catch the legs were nearly perpendicular to the ground.
\end{abstract}

Conclusions: The method allowed the measurement of the kinematic characteristics of the body during ergometer rowing. The measurements agreed with conventional technical teaching.

Clinical Relevance: Data collected on high-level rowers can provide a set of standard quantitative execution parameters that can be used by coaches as a benchmark for the assessment of technical movements in all rowers.

Keywords: Motion analysis, optoelectronic, rowing, sport, symmetry, three-dimensional.

\section{INTRODUCTION}

Rowing is an ancient human activity, where a shell (boat) is moved with the only help of the muscular strength of one or more oarspersons [1]. Rowing is a strenuous sport that stresses the body with a highly repetitive activity, where the "oarperson-shell-oar system" moves into the regatta basin water, an unstable and complex element [2-6]. Rowing movements involve both limb and trunk muscles, and requires well developed coordination and balance. During the years, different rowing techniques have been developed along with several modifications of the shell and oars $[3,6$, 7]. Anyway, a successful rowing technique requires a maximization of the horizontal direction of rowing (parallel to the water), allowing the largest part of the force to be actually used for propulsion [4, 8-10]. Body movements performed in directions different from the anterior-posterior

\footnotetext{
*Address correspondence to this author at the Dipartimento di Morfologia Umana e Scienze Biomediche "Città Studi", via Mangiagalli 31, I-20133 Milano, Italy; Tel: +39 - 02503 15407; Fax: +39-02 503 15387;

E-mail chiarella.sforza@unimi.it
}

one may increment the energetic cost, and produce balance alterations in both the shell and the body muscles and joints. The increased joint loads during the rowing cycle may generate overuse injuries (i.e., shoulder joint) or not-axial degenerative forces in the knee (meniscus overload). Thus, medium- and long-term pain and disability could occur $[2,8$, 10-13].

Rowing movements can also be simulated directly on the ground using specialized ergometers, that are widely used for body training $[2,8,10,12,14,15]$. Considering the difficulties for a global quantitative assessment of rowing performance directly in the regatta basin, ergometer rowing has become useful also for the assessment of body movements, forces, and energetic cost during simulated performances [2, 5, 6, 8-10,12-16].

Literature reports some recent studies on technical execution models, on spine movements and strengths developed during simulated rowing. Among others, the coupling of ergometers, electromagnetic and optoelectronic instruments, force transducers and magnetic resonance imaging have allowed the collection of data about spine 
movements and relevant forces expressed during simulated rowing, even in subjects with low back pain $[2,5,8,9,11$, 13, 14]. Nonetheless, three-dimensional data on body movements are still incomplete.

In our laboratory, a protocol for the three-dimensional quantitative analysis of body movements during ergometer rowing has been devised. The three-dimensional coordinates of body landmarks were obtained using a motion analyzer, and the range of motion of the head, spine, and lower limb angles, selected for their biomechanical and functional significance, was assessed to define normative data that take into consideration all body segments. Preliminary data on two rowers were previously reported [17]. In the current study, data on 18 high level rowers were obtained, and the movements of their head, spine, upper and lower limbs investigated.

The scope of the current investigation was twofold: first, we wanted to define a set of normative data that can be used by coaches and trainers during ergometer rowing, as well as within biomechanical models of rowing [6]; second, we wanted to assess if some of the kinematic variable identified during the rowing cycle had any relationship with anthropometric characteristics.

\section{MATERIALS AND METHODS}

\section{Participants and Experimental Set Up}

Eighteen high-level oarsmen were measured (Table 1). All rowers were (or had been) part of the National team, and they all had participated to national or international competitions with good or excellent results. They trained seven days a week, sharing their time between on water and off water exercises. In particular, they all were used to training on the ergometer.

They all were informed about the experimental procedures and possible risks, and signed an informed consent form that was previously approved by the local ethics committee, in accordance with the current laws. All procedures were not invasive, and did not involve risks different from those undertaken by the rowers during their daily off-water training.

Two primary rowing techniques exist: sculling and sweeping rowing. In sculling rowing each rower uses two oars, on separate sides of the shell, one for each hand; in sweeping rowing each rower uses one oar, held in both hands [18]. Considering that the analyzed oarsmen had a mixed experience of sweeping and sculling, and their familiarity with ergometer rowing, no subdivision of the sample was made following this criterium.
After some individually chosen warm-ups, each oarsman was asked to row on an ergometer (Concept 2 model) at 28 strokes/ min [4], performing 25-30 rowing cycles, with free split time (the time it takes to row $500 \mathrm{~m}$ ). The ergometer allows sculling style rowing. The three-dimensional movements of 21 body landmarks (left and right ankle, knee, greater trochanter, hip, shoulder, elbow, wrist, tragus; spinous process of $\mathrm{C} 7, \mathrm{~T} 2, \mathrm{~T} 12, \mathrm{~L} 2, \mathrm{~L} 4$; Fig. 1) were detected by an optoelectronic instrument (SMART System, B.T.S., Milan, Italy).

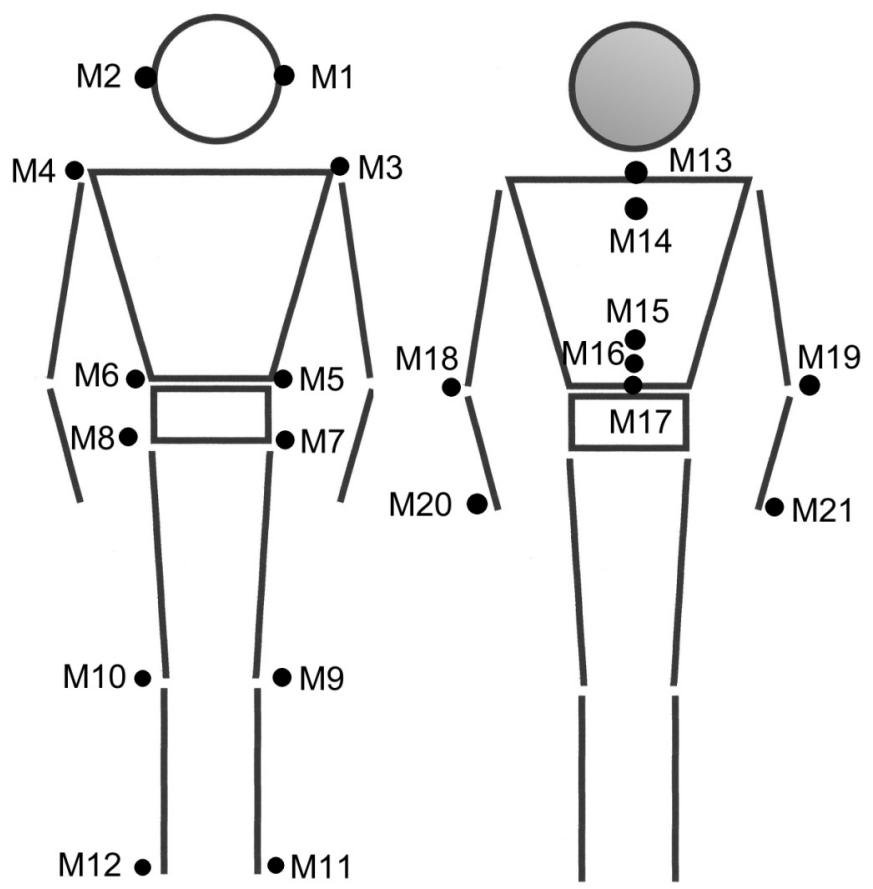

Fig. (1). Body landmarks. Paired (left and right): 1-2 tragi; 3-4 acromia; 5-6 superior iliac spines; 7-8 greater trochanters; 9-10 tibial tuberosities; 11-12 lateral malleoli; 18-19 radial heads; 20-21 ulnar styloid processes; Midline: 13 spinous process C7; 14 spinous process $\mathrm{T} 2 ; 15$ spinous process $\mathrm{T} 12 ; 16$ spinous process $\mathrm{L} 2 ; 17$ spinous process L4. Two further landmarks were positioned on the right and left fibular heads, and used for a static acquisition only.

The motion analyzer system detects the position of passive markers that are stroboscopically illuminated by infrared light (IR light length, $730 \mathrm{~nm}$ ) using nine infraredsensitive charge-coupled (CCD) TV cameras with a $659 \mathrm{x}$ 490 pixel matrix: the cameras were positioned at a variable height from the floor, at various angles of a working volume of $200 \times 150 \times 250 \mathrm{~cm}$ to film each oarsman from different points of view. A sampling rate of $120 \mathrm{~Hz}$ was used. The cluster of TV cameras was previously calibrated with an

Table 1. Analyzed Rowers

\begin{tabular}{|c|c|c|c|c|c|c|c|c|}
\hline & Age (y) & Height (m) & Body Mass (kg) & Head Width (m) & Knee Width (m) & BMI (kg/m $\mathbf{m}^{2}$ & Experience (y) & Training (Hours/Week) \\
\hline \hline Mean & 22.1 & 1.83 & 78.1 & 0.17 & 0.35 & 23.22 & 7.4 & 28 \\
\hline SD & 2.5 & 0.06 & 8.1 & 0.38 & 0.02 & 1.44 & 2.6 & 8 \\
\hline Min & 19 & 1.74 & 68 & 0.13 & 0.40 & 20.45 & 3 & 14 \\
\hline Max & 27 & 1.95 & 98 & 0.24 & 0.31 & 26.31 & 12 & 35 \\
\hline
\end{tabular}

Head width is measured between right and left tragi. 
accuracy of $0.01 \%(1 / 10000$ of the maximal dimension of the useful acquisition volume) $[19,20]$.

During the execution of the movements, the instrument software (SMART Tracker, B.T.S., Milan, Italy) recognized the coordinates of each marker for any TV camera. Subsequently, all the coordinates were converted to real metric data, and a set of $x, y, z$ coordinates for each landmark in each frame that constituted the movement was obtained $[21,22]$.

For the current experiment, $10 \mathrm{~mm}$ spherical markers were used. An additional marker was positioned on the horizontal part of the ergometer to provide a reference. The trial was repeated three times for each rower, and only data collected during the central ten strokes (11th-20th stroke) were further analyzed. Between each trial, the rowers were allowed to rest, ensuring that the tests were performed all in the same conditions.

\section{Data Analysis}

Dedicated software, developed by one of the Authors (VFF), was used to compute several angles and distances to characterize head, spine, upper and lower limb movements during rowing.

In particular, the dimension and range of motion of the posterior angles of the cervical (inter tragi to $\mathrm{C} 7$ ), thoracic (T2-T12) and lumbar (L2-L4) spine segments (Fig. 2) relative to the horizontal axis were computed in the sagittal plane (unit: degrees) [13]. Head inclinations in the horizontal and frontal planes was measured in $\mathrm{mm}$, and expressed as a percentage of head width (inter tragi distance, Fig. 3).

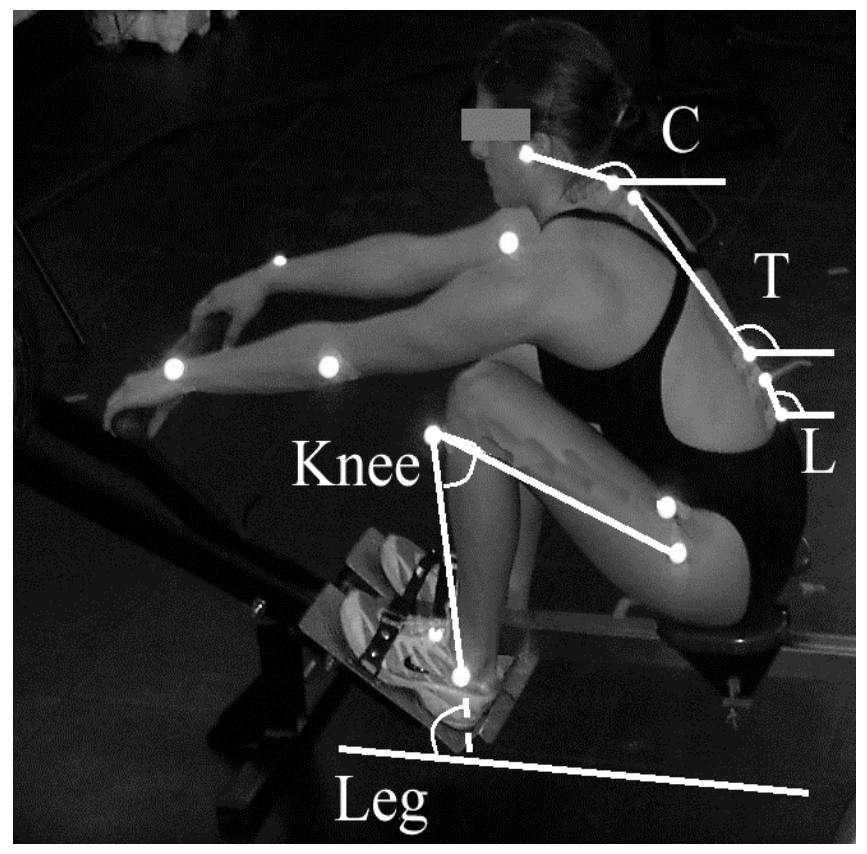

Fig. (2). Sagittal plane angles computed to analyze spine and lower limb movements. L: lumbar; $\mathrm{T}$ : thoracic; $\mathrm{C}$ : cervical spine segments; knee and leg angles are also shown.

To assess limb symmetry, knee movements (distance between right and left knee, unit: $\mathrm{mm}$ ) and shoulder, elbow and wrist movements (absolute differences of the right-left marker positions in the anterior-posterior and vertical directions, unit: $\mathrm{mm}$ ) were analyzed (Fig. 3).

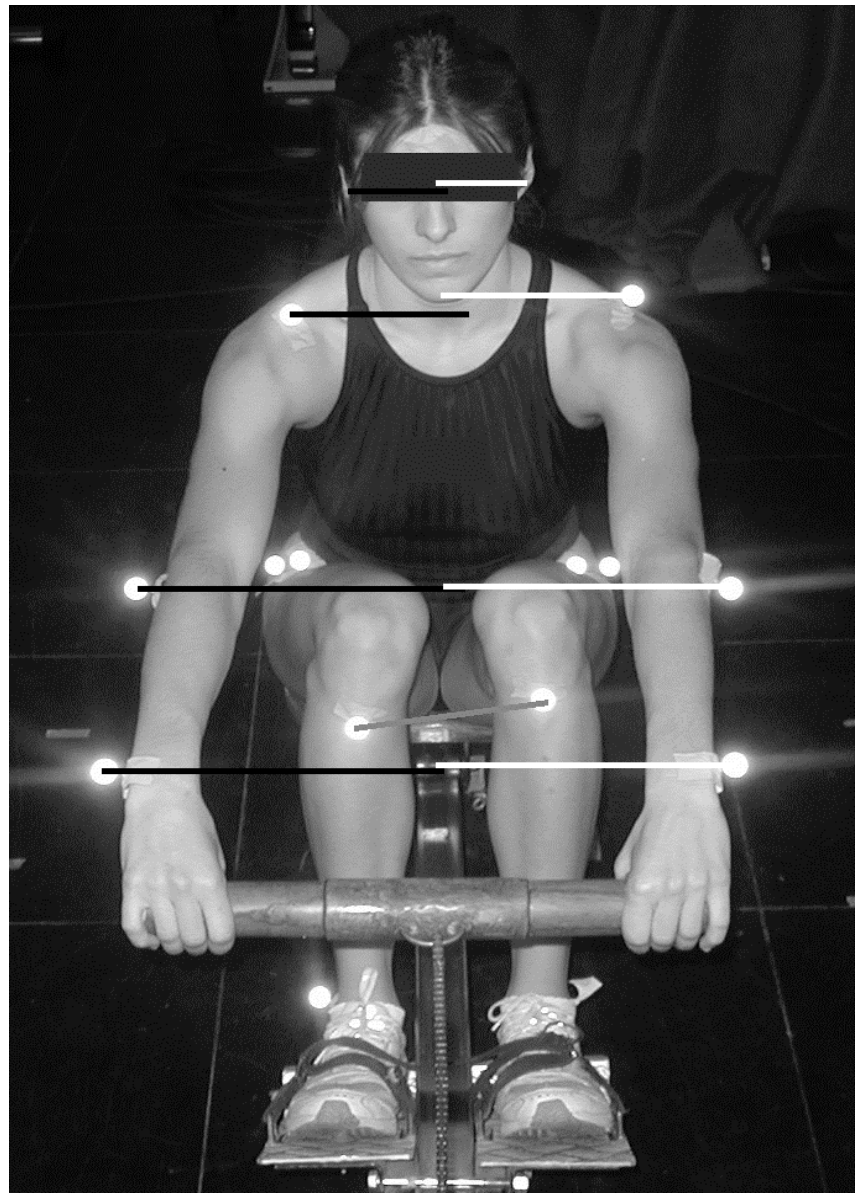

Fig. (3). Frontal plane symmetry assessment: knee (grey line, distance between right and left knee markers), tragi, shoulder, elbow and wrist (absolute differences between the right -black lines- and left -white lines- marker positions).

Lower limb movements were assessed by calculating leg position $v s$ the ground (knee-ankle line vs the ground, unit: degrees), and knee angle (greater trochanter- knee- ankle angle, unit: degrees). As detailed by Lovecchio et al., [17], knee landmark position (tibial tuberosities) was corrected using a further static acquisition where two additional anatomical markers (fibular heads) were also positioned [19]. Lower limb angles were computed in the sagittal plane, pooling right and left side (Fig. 2). A preliminary control assessed if the movement of the lower limbs was all performed within a sagittal plane. This was made by computing the mediolateral displacements of the lower limb landmarks, and of lower limb lengths along the rowing cycle.

Head, spine and lower limb data were obtained at catch and finish: lower limb position (knee extension and leg position $v s$ the ground) was used to automatically detect catch (minimal knee extension) and finish (maximal knee extension). Upper limb data were computed as differences between the right- and left-side relevant landmarks along all the rowing cycle.

For each trial, angular statistics was used to obtain mean values and relevant standard deviations across the ten strokes that were averaged for each oarsman. The three trials (result of the average of 10 strokes) were used to compute intrarower variability, and then averaged within each participant. 
Descriptive statistics were computed for the analyzed rowers; linear regression analyses were run between individual and anthropometric characteristics (age, training, body dimensions), and distances and angles obtained during the rowing trials. A p value of 0.01 or less was considered significant.

\section{RESULTS}

In the 18 analyzed rowers, stroke rate ranged between 23.9 and 31 strokes/ min (mean, 27.5; SD, 0.8), in good accord with the protocol. Individual variations in stroke rate were limited, with SD computed over the three trials ranging between 0.5 and 1.9 strokes/ min.

A preliminary control verified that lower limb movements were performed entirely in the sagittal plane, without out of plane movements. In all occasions, the average medio-lateral landmark displacements were smaller than marker dimensions for hip and ankle landmarks, and somewhat larger for knee landmark (on average, $9.4 \mathrm{~mm} \mathrm{SD}$ 3.4 ; greater trochanter right, $7.4 \mathrm{~mm}$, left $9.6 \mathrm{~mm}$; knee, right $13.5 \mathrm{~mm}$, left $13.3 \mathrm{~mm}$; ankle right $6.9 \mathrm{~mm}$, left $5.7 \mathrm{~mm}$ ). Also, the leg (knee-ankle) and thigh (greater trochanterknee) lengths did not change during the rowing cycle (coefficients of variation ranging between 2.4 and 3.3\% mean 2.9, SD 0.4). Therefore, pooled right-left lower limb angles were analyzed in the sagittal plane.

Mean and standard deviations of spine, head and limb movements measured during ergometer rowing in all 18 participants are shown in Table 2.

Head inclination and rotation were measured in $\mathrm{mm}$ and expressed as a percentage of head width $(\mathrm{t}-\mathrm{t})$.

All rowers performed a complete extension of their lower limbs during the ergometer rowing. On average, at catch they reached an angle close to $90^{\circ}$ for the leg position (ankle-knee $v s$ ground), ranging between 78 and $99^{\circ}$ (Table 2). At finish,

Table 2. Spine, Head and Limb Movements During Ergometer Rowing

\begin{tabular}{|c|c|c|c|c|c|}
\hline & & Unit & Catch & Finish & ROM/ R-L Difference \\
\hline \multicolumn{6}{|l|}{ Spine } \\
\hline Head/ neck & $\begin{array}{c}\text { Mean } \\
\text { SD }\end{array}$ & Deg & $\begin{array}{c}163.5 \\
5.9\end{array}$ & $\begin{array}{c}140.7 \\
8.2\end{array}$ & $\begin{array}{c}22.8 \\
8.9\end{array}$ \\
\hline Thoracic & $\begin{array}{c}\text { Mean } \\
\text { SD }\end{array}$ & Deg & $\begin{array}{c}138.2 \\
4.6\end{array}$ & $\begin{array}{c}70.4 \\
6.3\end{array}$ & $\begin{array}{c}67.8 \\
5.5\end{array}$ \\
\hline Lumbar & $\begin{array}{c}\text { Mean } \\
\text { SD }\end{array}$ & Deg & $\begin{array}{c}109.0 \\
14.2\end{array}$ & $\begin{array}{c}49.8 \\
7.9\end{array}$ & $\begin{array}{l}59.3 \\
15.4\end{array}$ \\
\hline \multicolumn{6}{|l|}{ Head position } \\
\hline Inclination & $\begin{array}{c}\text { Mean } \\
\text { SD }\end{array}$ & $\%$ & $\begin{array}{l}5.20 \\
4.64\end{array}$ & $\begin{array}{l}4.99 \\
5.06\end{array}$ & -- \\
\hline Rotation & $\begin{array}{c}\text { Mean } \\
\text { SD }\end{array}$ & $\%$ & $\begin{array}{c}9.66 \\
10.69\end{array}$ & $\begin{array}{l}11.20 \\
14.97\end{array}$ & -- \\
\hline \multicolumn{6}{|l|}{ Upper limbs } \\
\hline Shoulders vertical & $\begin{array}{c}\text { Mean } \\
\text { SD }\end{array}$ & $\mathrm{mm}$ & -- & -- & $\begin{array}{c}10 \\
7\end{array}$ \\
\hline Shoulders anteroposterior & $\begin{array}{c}\text { Mean } \\
\text { SD }\end{array}$ & $\mathrm{mm}$ & -- & -- & $\begin{array}{c}11 \\
7 \\
\end{array}$ \\
\hline Elbows vertical & $\begin{array}{c}\text { Mean } \\
\text { SD }\end{array}$ & $\mathrm{mm}$ & -- & -- & $\begin{array}{l}17 \\
12 \\
\end{array}$ \\
\hline Elbows anteroposterior & $\begin{array}{c}\text { Mean } \\
\text { SD }\end{array}$ & $\mathrm{mm}$ & -- & -- & $\begin{array}{l}20 \\
13 \\
\end{array}$ \\
\hline Wrists vertical & $\begin{array}{c}\text { Mean } \\
\text { SD }\end{array}$ & $\mathrm{mm}$ & -- & -- & $\begin{array}{l}18 \\
12\end{array}$ \\
\hline Wrists anteroposterior & $\begin{array}{c}\text { Mean } \\
\text { SD }\end{array}$ & $\mathrm{mm}$ & -- & -- & $\begin{array}{l}16 \\
12 \\
\end{array}$ \\
\hline \multicolumn{6}{|l|}{ Lower limbs } \\
\hline R-L Knee distance & $\begin{array}{c}\text { Mean } \\
\text { SD }\end{array}$ & $\mathrm{mm}$ & $\begin{array}{c}323 \\
87\end{array}$ & $\begin{array}{c}212 \\
68\end{array}$ & $\begin{array}{c}112 \\
38\end{array}$ \\
\hline Knee angle & $\begin{array}{c}\text { Mean } \\
\text { SD }\end{array}$ & Deg & $\begin{array}{c}63.4 \\
3.3 \\
\end{array}$ & $\begin{array}{c}170.1 \\
4.7\end{array}$ & $\begin{array}{c}-106.8 \\
5.7 \\
\end{array}$ \\
\hline Leg $v s$ ground & $\begin{array}{c}\text { Mean } \\
\text { SD }\end{array}$ & Deg & $\begin{array}{c}91.5 \\
6.2 \\
\end{array}$ & $\begin{array}{c}16.8 \\
2.4\end{array}$ & $\begin{array}{c}74.6 \\
6.7\end{array}$ \\
\hline
\end{tabular}


their knee angle was on average close to $180^{\circ}$ (complete extension), ranging between 160 and $178^{\circ}$ (Fig. 4). Both angles were well repeatable within participant over the three trials, with standard deviations comprised between $0.06^{\circ}$ (knee angle and leg angle $v s$ the ground at finish) and $3.42^{\circ}$ (knee angle at catch).

At catch, the inter-knee distance was very similar to its rest value (maximum value), at finish it was approximately $40 \%$ smaller than at rest (minimal distance), with an average excursion of $112 \mathrm{~mm}$.

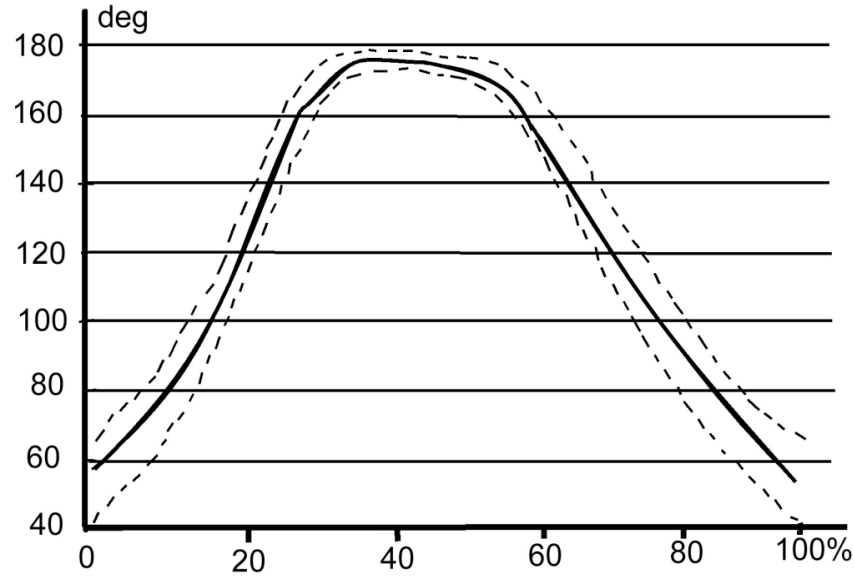

Fig. (4). Knee angle during the rowing stroke, mean and standard error. The time base has been normalized with respect to movement time for each of the analyzed rowing strokes.

Within each participant, the three trials were made with similar spine angles, with reduced individual coefficients of variation, especially at catch and in the more cranial segments of the spine (mean values: head/ neck, catch $0.45 \%$, finish $0.57 \%$; thoracic spine, catch $0.44 \%$, finish $1.54 \%$; lumbar spine, catch $0.61 \%$, finish $2.8 \%$ ).

The head and neck were approximately parallel to the horizontal at catch (inter tragus-C7 vs horizontal $164^{\circ}$ ), and they extended at finish $\left(141^{\circ}\right)$. During the rowing cycle, the head had limited horizontal (rotation, 10\% of intertragi distance at catch, $11 \%$ at finish) and frontal plane (inclination, 5\% at both catch and finish) inclinations. The thoracic spine (T2-T12 vs horizontal) extended on average of $68^{\circ}$ during the stroke. The average range of motion of the lumbar spine (L2-L4 vs horizontal) was $59^{\circ}$.

Linear regression analyses were run between anthropometric characteristics and distances and angles obtained during the rowing trials, but only those that were statistically significant $(\mathrm{p}<0.01)$ are reported. Overall, weightier oarsmen had lower ranges of motion in the thoracic $(\mathrm{r}=-0.661, \mathrm{p}=0.003)$ spine segments. A similar negative correlation was found with the body mass index $(\mathrm{r}=$ -0.752, p $<0.001$ ), while no correlation was found with standing height $(\mathrm{r}=-0.335, \mathrm{p}=0.174)$.

The upper limbs were symmetric during the analyzed ergometer rowing, with absolute average right-left differences up to $20 \mathrm{~mm}$ in both the vertical and anteriorposterior planes. Throughout the movement, also considering marker dimensions $(10 \mathrm{~mm})$, the movement can be judged symmetric. In the anterior-posterior plane, all three right-left differences were significantly correlated to standing height (wrist: $\mathrm{r}=0.671, \mathrm{p}=0.0023$; elbow: $\mathrm{r}=0.717, \mathrm{p}<0.001$; shoulder: $\mathrm{r}=0.635, \mathrm{p}=0.005)$.

\section{DISCUSSION}

Optoelectronic instruments are currently considered the best method for three-dimensional motion capture during both sport performance and body movements, minimally interfering with the movement execution $[5,8,10,16,19-$ 24]. Also, usually these instruments have no interferences with other laboratory tools [19]. For instance, several previous investigations on rowing movements were performed using electromagnetic devices [2, 8, 11, 12], but it has recently reported that these instruments may interfere with the ferrous components of the ergometer [25].

Among the studies that used optical instruments, Caldwell et al., [13] used a two-dimensional TV camera system to record lumbar spine motion in a single plane, while Halliday et al., [5] used a three-dimensional motion analyser to detect a complete body model, but they assessed only five oarsmen. Hofmijster et al., [9] positioned the landmarks only on the left side of the body, and reported data only on the center of mass of their oarswomen. A more detailed analysis was made by Pollock et al., [10] on nine oarswomen, but they reported only data on spinal motion as related to muscle activity.

The protocol used in the current study allowed the measurement of selected kinematic characteristics of the body during ergometer rowing. Segmental spine movements were analyzed as suggested in previous studies for both their importance in rowing performance, and their possible relationship with back pain $[2,8,12-14]$. Care was taken to avoid fatigue during the performance, allowing a complete recovery between the three trials. Indeed, fatigue has been reported to increase lumbar flexion and limit femoral extension during simulated rowing, thus changing movement performance $[2,13]$. Also, an almost constant stroke rate was maintained, because previous studies reported a reduction in the rotation of the lumbar spine at increasing rates [8].

Ranges of motion of the trunk and timing of maxima and minima were in good accord with literature data $[2,8,12-$ 14], while no previous investigation reported data on head and neck motion during rowing. In contrast, this part of the body of the rower has been considered in the most recent biomechanical models [6]. During boat rowing, the rower should used vision to control his trajectory, but during ergometer rowing this was not necessary. We failed to recommend to our rowers to maintain a fixed visual target during the experiment, and this should be considered a limitation of the study. Therefore, the direct transfer of these data to actual skull rowing should be attentively considered. Nonetheless, asymmetrical head movements may influence spine motion, with subsequent asymmetric distributions of loads between the limbs, and they should be avoided.

In the current investigation, weightier oarsmen were found to have lower ranges of motion in their thoracic spine segment. This finding is of difficult interpretation, because no previous literature reports of this kind were found. A possible explanation may be that rowers with an increased body mass could move less in general. Unfortunately, we did not assess the actual range of motion of the various body and spine segments, and cannot express these data as percentages 
of the maximum possible movement $[13,21,24]$. This assessment, together with multiple correlation analyses among the analyzed variables, may offer a deeper insight into the individual characteristics of ergometer rowing. No significant relationship was found between standing height and spine movements, but upper limb asymmetry also depended on body dimensions.

Upper limb symmetry during rowing was assessed because our oarsmen came from various experiences of sculling and sweeping, and we wanted to see if they could maintain a good level of symmetry on the ground (off water). Indeed, most of biomechanical simulations modelled rowing within symmetric situations $[3,6,15]$. Even if this can be a good approximation for the lower limbs, as found in the current study, its actual effect on the upper limbs has never been tested. Significant correlations between body dimensions and the right-left differences of the analyzed upper limb landmarks were found. Even if the limited asymmetries may have a minor effect on actual rowing performance, they should be considered when arranging rowing crews [4], and they may affect general equilibrium and balance. Indeed, during rowing the upper limbs are those that have the largest "contact" with the environment (via the oars). Their functional asymmetries may also be a way to cope with possible asymmetries in the regatta basin, thus permitting to the rowers to correct the shell trajectory with a minimal energy loss.

Limited data about lower limb movements were found in literature $[8,12]$. Current values were in good accord with the technical descriptions by coaches and those used in biomechanical simulations during rowing $[3,15]$. At catch, the leg should be nearly vertical $v s$ the ground (full knee flexion), becoming nearly horizontal at finish (full knee extension), thus allowing the complete sliding movement of the seat during the rowing cycle. In all the current rowers, the recorded values were in good agreement with this pattern. A full lower limb (and seat) motion will permit a complete action of the upper limbs- oars system [6, 15]. Indeed, in no rower a knee angle of 180 degrees was recorded, but considering marker dimensions, the current values can be considered a good approximation [12], and were even larger than those reported by Halliday et al., [5]. Further investigations may assess the effect of different rowing rates [8], because with an increased rowing rate there may not be sufficient time for a complete knee extension, especially for the less experienced rowers. In elite oarsmen, Halliday et al., [5] reported little differences in knee extension with rowing rates ranging between 20 and 32 strokes/ min. In contrast, in collegiate rowers, McGregor et al., [8] reported a reduction in leg extension and an increase in femoral flexion (hip flexion) with rowing rates ranging between 17 and 36 strokes/ $\mathrm{min}$.

Literature reports on a successful rowing performance mostly dealt with metabolic (aerobic capacity) and anthropometric (body fat mass) characteristics [1], but biomechanical aspects cannot be neglected $[2-4,9,13]$. Nonetheless, the effect of body movements during rowing has been scanty analyzed. Hofmijster et al., [9] found a relationship between performance and rowing skill, but reported only global data about the centre of mass of their rowers. In canoeists, Rodano et al., [16] found that elite performers had wider joint ranges of motion than intermediate and novice performers. As suggested by Halliday et al., [5], we choose to assess only high-level (elite) rowers because they are very consistent, as shown by the reduced inter-individual SD found in the current study, that well parallels previous findings about force production [4]. Indeed, data obtained from elite rowers can be used as standards to further compare novice rowers, thus providing a quantitative technical support to trainers and coaches $[10,12$, 14].

A limitation of the current study is that we did not collect performance data about our oarsmen. Nonetheless, their anthropometric characteristics well paralleled those of the rowers with the best rowing performance analyzed by Yoshiga and Higuchi [1]. Also, we did not investigate all possible anthropometric variables, and future investigations may possibly assess spinal movements with a greater detail.

Further studies could identify useful kinematic parameters to screen the movements in subjects with muscular-skeletal injuries [2, 11], even trying to detect "malpractice" before the development of damages. Current data could also be used inside biomechanical models of rowing, where also the shell and the regatta basin fluid movements can be considered [6].

\section{ACKNOWLEDGEMENTS}

Declared none.

\section{CONFLICT OF INTEREST}

Declared none.

\section{REFERENCES}

[1] Yoshiga C, Higuchi M. Rowing performance of female and male rowers. Med Sci Sports Exerc 2003;13: 317-22.

[2] Bull AM, McGregor AH. Measuring spinal motion in rowers: the use of an electromagnetic device. Clin Biomech 2000; 15: 772-6.

[3] Baudouin A, Hawkins D. A biomechanical review of factors affecting rowing performance. Br J Sports Med 2002; 36: 396-402.

[4] Baudouin A, Hawkins D. Investigation of biomechanical factors affecting rowing performance. J Biomech 2004; 37: 969-76.

[5] Halliday SE, Zavatsky AB, Hase K. Can functional electric stimulation-assisted rowing reproduce a race-winning rowing stroke? Arch Phys Med Rehabil 2004; 85: 1265-72.

[6] Formaggia L, Miglio E, Mola A, Montano A. A model for the dynamics of rowing boats. Int J Numer Meth Fluid 2009; 61: 11943.

[7] Caplan N, Gardner TN. Optimization of oar blade design for improved performance in rowing. J Sports Sci 2007; 25: 1471-8.

[8] McGregor A, Bull A, Byng-Maddick R. A comparison of rowing technique at different stroke rates: a description of sequencing, force production and kinematics. Int J Sports Med 2004; 25: 46570.

[9] Hofmijster MJ, Van Soest AJ, De Koning JJ. Rowing skill affects power loss on a modified rowing ergometer. Med Sci Sports Exerc 2008; 40: 1101-10.

[10] Pollock CL, Jenkyn TR, Jones IC, Ivanova TD, Garland SJ. Electromyography and kinematics of the trunk during rowing in elite female rowers. Med Sci Sports Exerc 2009; 41: 628-36.

[11] McGregor A, Anderton L, Gredoyc W. The assessment of intersegmental motion and pelvic tilt in elite oarsman. Med Sci Sports Exerc 2002; 34:1143-9.

[12] McGregor AH, Patankar ZS, Bull AM. Spinal kinematics in elite oarswomen during a routine physiological "Step Test". Med Sci Sports Exerc 2005; 37: 1014-20.

[13] Caldwell J, McNair P, Williams M. The effect of repetitive motion on lumbar flexion and erector spinae muscle activity in rowers. Clin Biomech 2003; 18: 704-11. 
[14] O'Sullivan F, O'Sullivan J, Bull A, McGregor A. Modelling multivariate biomechanical measurements of the spine during a rowing exercise. Clin Biomech 2003; 18: 488-93.

[15] Consiglieri L, Pires EB. An analytical model for the ergometer rowing: inverse multibody dynamics analysis. Comput Methods Biomech Biomed Engin 2009; 12: 469-79.

[16] Rodano R, Squadrone R, Sacchi M, Marzegan A. A 3D kinematic analysis of canoeing on a simulator: differences between elite, intermediate and novice canoists. In: Blackwell JR, Ed. Proceedings of oral session XIX I.S.B.S. San Francisco (CA): University of San Francisco 2001, pp. 267-71.

[17] Lovecchio N, Casiraghi E, Pirola C, Shirai YF, Sforza C, Ferrario VF. A three-dimensional study of trunk and lower limb motion during ergometer rowing. Preliminary data. In: Starosta W, Squatrito S, Eds. Sport Kinetics 2005. Scientific fundaments of human movement and sport practice. Bologna (I): Centro Universitario Sportivo Bolognese 2005; p. 2.418-2.9.

[18] Elliott B, Lyttle A, Birkett O. The RowPerfect ergometer: a training aid for on-water single scull rowing. Sports Biomech 2002; 1: $123-34$
[19] Chiari L, Della Croce U, Leardini A, Cappozzo A. Human movement analysis using stereophotogrammetry. Part 2: instrumental errors. Gait Posture 2005;21:197-211.

[20] Sforza C, Rango M, Galante D, Bresolin N, Ferrario VF. Spontaneous blinking in healthy persons: an optoelettronic study of eyelid motion. Ophthalmic Physiol Opt 2008; 28: 345-53.

[21] Tommasi DG, Foppiani AC, Galante D, Lovecchio N, Sforza C. Active head and cervical range of motion: effect of age in healthy females. Spine 2009; 34: 1910-6.

[22] Sforza C, Corradini C, Grassi GP, et al. Cervical range of motion in rugby league players. Open Sports Med J 2010; 4: 114-8.

[23] Grassi G, Turci M, Shirai YF, Lovecchio N, Sforza C, Ferrario VF. Body movements on the men's competition mushroom: a three dimensional analysis of circular swings. Br J Sports Med 2005; 39: 489-92.

[24] Baudry L, Sforza C, Leroy D, Lovecchio N, Gautier G, Thouvarecq R. Amplitude variables of circle on the pedagogic pommel horse in gymnastics. J Strength Cond Res 2009; 23: 705-11.

[25] Ng L, Burnett A, Campbell A, O'Sullivan P. Caution: the use of an electromagnetic device to measure trunk kinematics on rowing ergometers. Sports Biomech 2009; 8: 255-9. 